\title{
POWER SUPPLY FOR LUMINAIRES LOCATED IN RESTRICTED AREAS WITH PHOTOVOLTAIC PANELS CALCULATING MPPT
}

\author{
P. ONU \\ Doctoral School of Engineering Sciences, Aleea Sinaia, nr. 13, 130004 Targoviste, Dambovita, Romania \\ E-mail: petru_onu@yahoo.com
}

\begin{abstract}
The proposed system is a method to power supply luminaires in inaccessible areas. It proposes a method with using PV panels and calculating MPPT. It is a method of supply luminaires with $\mathrm{LiFePO4}$ batteries using remote transmission and transmission electrical parameters using microcontrollers for a complete autonomy and management system.
\end{abstract}

Keywords: photovoltaic panel, MPPT, $\mathrm{LiFePO}_{4}$ battery, SolidState Lighting, Wi-Fi, GPRS

\section{INTRODUCTION}

Lighting is one of the most important element in the development of a city or some places where we need lighting for both indoor and outdoor. If cities or entities of housing don't have access to the national grid of electricity, there is problem concerning their development. However, a problem occurs where we need lighting in remote or restricted areas, where is no infrastructure for lighting or simply a problem is in its positioning. In these cases, it needs to find solutions for supplying with electricity from other energy sources.

Once chosen option for power supply, we need solutions for storing this energy because there it always need for electricity while it is therefore to be analyzed possibilities for energy storage for possible use thereof.

After the first two steps were made, now we must find a solution for the management and control of stored energy, as well as a mechanism that could take energy from the power supply and store in a storage sources previously chosen.

Finally, we will study methods of management and control and to take various sources of power supply, from the consumer and the environment in which they are found.

Street lighting solutions, some existing on the market, in most cases are using LED lamps powered by solar panels and whose control is performed by light sensors. Complex solutions uses remote management, control technology and data transmission on wired or wireless network. For inaccessible or isolated areas, such of these solutions can be useful and experience in this segment can be successfully employed.
In such systems, street lighting poles or lighting inscriptions that have long distances from the station, must communicate with each other using a communication protocol, and between all of the system and station should be used another protocol, which complicates less given system architecture.

The research in the scientific literature were directed to lighting solutions using LED [1] and for the control and management solutions are using:

- coupled wired modules combined with wireless solutions, here given PLC (Power Line Communication) and GPRS (General Packet Radio Service) [2] or RS485, ZigBee, GPRS [3];

- wireless sensors [4], [5] technology as Wi-Fi but on different segments being controlled through GPRS;

- which allow minimizing system power consumption and computing device transmitter / receiver as XBee technology given here, ZigBee [6], [7];

- $\quad$ that transport information using wireless devices as nRF24L01[8], [9], [10].

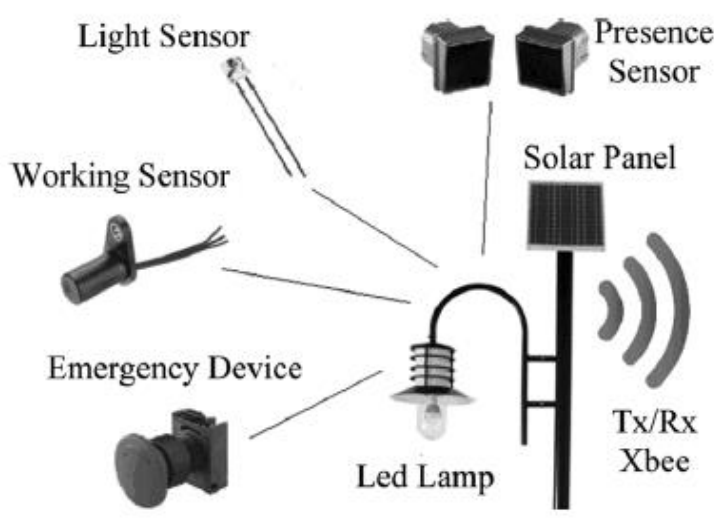

Figure 1. Solution for street lighting [11]

Each pole will have one photovoltaic panel, a battery and a microcontroller that will charge the battery and communicate with another pole, thus forming a local network. This network we deployed is using a Wi-Fi module for each pole, all modules communicate with each other forming a small network in a specific point is connected to a concentrator. He takes all the data from each body part and through a GPRS module will transfer the data to a control center that is located remotely. Such an operator in any time can see what state is each module, consumption, temperature, errors and other notifications. 


\section{DEVICES AND WORKING METHODS}

To begin we must analyze the environment where we require power supply. For this we must define the following types of areas where they have to be implemented such a system.

We must choose different types of supply for different areas needed, such as the following types of restricted areas:

- In terms of administrative (infrastructure);

- In terms of maintenance;

- In terms of the area of operation;

- In terms of the environment (they are divided into several types: explosive, toxic, polluting and mining).

As a first model we will create a system for power supply in an area difficult to access from the administrative point of view, which is an isolated area where there is no network of electrification. So, necessity of creating a power supply system increases significantly.

\subsection{Supply with power}

For these systems we resort to the methods of the renewable resources of power already known, but here we need to research the area to find the best and most effective ways from power supply that we can find in the immediate vicinity.

From this, the most common methods of power supply of inaccessible areas from administrative point of view, we can find solutions that:

- Power sources based on wind energy;

- Power sources based on photovoltaic panels;

- Power sources based on hydropower;

- Power sources based on both hydropower and wind power, designed as autonomous system.

\subsection{Communications}

Choosing a power supply will be carried out exclusively under supply opportunities in the area where we want to achieve lighting. Thus the most convenient, cheap and safe system is based on photovoltaic panels.

To always have access to all data regarding the luminaires, their current state, consumption and other characteristics must be considered better situation where we have implemented this system to choose the mode of communication that we will choose.

Possibilities of communication presented at present we will mention as follows:

- Power Line Communication (PLC);

- Controller Area Networks communication;

- Bluetooth communication;

- Communication via Wi-Fi;

- Radiocommunication via nRF24L01;
- Xbee, ZigBee communication.

For communication in our case, a system into an area difficult to access, exemplifying be at a greater height, where the national power grid has not reached, must choose a type wireless, where communication of PLC and CAN we can't use.

Therefore, we'll analyze other types of communication to find the solution to use. A small characterization of each method is presented in Table 1 .

Table 1 Differences between communications technologies

\begin{tabular}{|c|c|c|c|}
\hline Standard & ZigBee & $\begin{array}{c}\text { Wi-Fi'M } \\
\mathbf{8 0 2 . 1 1 b}\end{array}$ & $\begin{array}{c}\text { Bluetooth } \\
\mathbf{8 0 2 . 1 5 . 1}\end{array}$ \\
\hline $\begin{array}{c}\text { Transmission } \\
\text { Range (meters) }\end{array}$ & $1-100^{*}$ & $1-100$ & $1-10$ \\
\hline $\begin{array}{c}\text { Battery Life } \\
\text { (days) }\end{array}$ & $100-1,000$ & $0.5-5.0$ & $1-7$ \\
\hline $\begin{array}{c}\text { Network Size (\# } \\
\text { of nodes) }\end{array}$ & $>64,000$ & 32 & 7 \\
\hline $\begin{array}{c}\text { Stack Size (KB) } \\
\text { Throughput } \\
\text { (kb/s) }\end{array}$ & $4-32$ & 1,000 & 250 \\
\hline Cost & High & Low & Free \\
\hline
\end{tabular}

Bluetooth is a good solution but not allowing interconnection of several devices simultaneously and it is more vulnerable from terms of the attacks therefore we can't use it. What would fit is a way for networking via Wi-Fi. It has a lower price and the transfer rate, higher than in a device with Bluetooth.

\subsection{Battery}

For storing energy from source (photovoltaic panel this case) we require batteries. The efficiency of this system increases with choosing a correct battery, which meets our requirements. Therefore, we must analyze all types of batteries and use the most efficient from all points of view.

In the past years interest to lead-acid batteries to store energy in such system gradually decreased due to the negative effects they play. I will present some differences, advantages and disadvantages, and choice of the correct battery for this system.

Lead-Acid batteries:

- Limited "useable" capacity (30-50\%);

- $\quad$ Limited life cycles (350-500);

- High maintenance costs;

- Their high weight;

- The charging is slower;

- Temperature imbalance shortens the battery life, even may damage completely; 
These are the reasons why we can't use this type of battery.

To solve this problem, I will choose a Li-Ion battery technology. This type of battery it is widely used for different applications, with some pluses and some minuses and with reference to a particular application. Several types from technology are:

- Lithium-Cobalt-Oxide $\left(\mathrm{LiCoO}_{2}\right)$;

- $\quad$ Lithium Nickel Oxide $\left(\mathrm{LiNiO}_{2}\right)$;

- Lithium Manganese Oxide $\left(\mathrm{LiMn}_{2} \mathrm{O}_{4}\right)$;

- Lithium NMC ( $\left.\mathrm{Li}(\mathrm{LiaNixMnyCoz}) \mathrm{O}_{2}\right)$;

- Lithium Iron-Phosphate $\left(\mathrm{LiFePO}_{4}\right)$;

In case of storage energy generated by photovoltaic panels, the best type of battery is LiFePO4. This type of batteries has the following advantages:

- High efficiency for charging and discharging;

- Employable capacity rising to over $85 \%$ of the total battery capacity;

- Charging the battery in less time (it can be loaded forced a period of time without decreasing efficiency);

- Is approximately $30 \%$ lighter than the lead-acid battery;

- Much larger life cycles (3000-5000 cycles).

\section{DETAILS AND OPERATION MODE}

This system can be extremely effective if it considers many parameters that can improve the efficiency and effectiveness. Therefore, I will analyze several factors to be considered.

The efficiency of a photovoltaic panel of good quality still has a efficiency below $15 \%$, this is why we need to calculate to use all the energy that can be produced from it. Classical methods of charging a battery from the photovoltaic panel representing in itself the charging unattended, which led to a decrease in its efficiency by charging voltage and current given by the photovoltaic panel at a time, so you should find a solution to use the largest photovoltaic panel power at any point of time.

This process is tracing maxim power point of PV panel. Therefore I will implement an algorithm that will be implemented in a microcontroller which then will also serve for further communication via a communication channel. Block diagram of system with MPPT schematically is represented in (Figure 2). It describes the operating principle and calculation maxim power point.

This mechanism will check permanent input voltage and input current and make a comparison if the power input is higher when given last time when this calculated power and voltage is greater than the last time he measured it then microcontroller will generate a signal that will increase voltage, this procedure will perform until when power will be calculated $\mathrm{P}(\mathrm{k})<\mathrm{P}(\mathrm{k}-1)$ when the microcontroller will decrease the intensity of the input voltage.

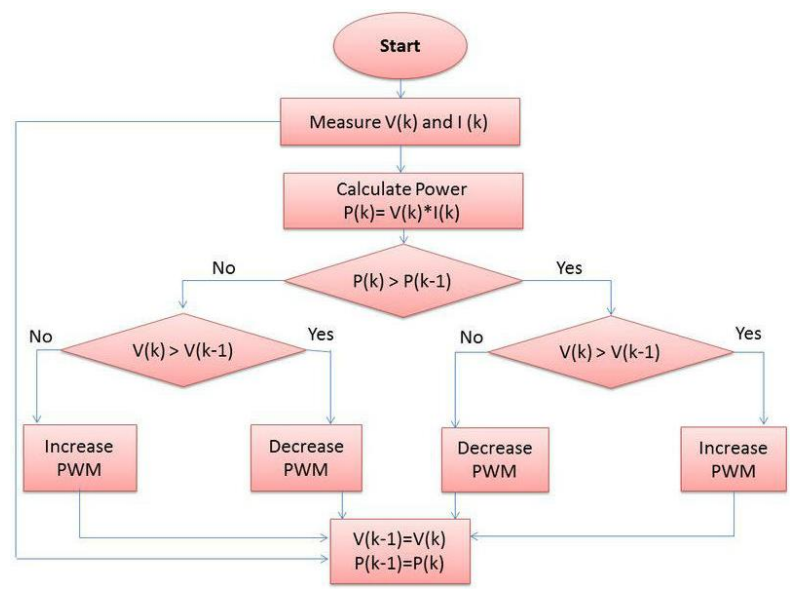

Figure 2. Block diagrame of sistem

In this way the microcontroller will follow as always power input to be one of the highest value (according to Figure 3) where the red line is marked calculated output and the next image (Figure 4) is plotted result ago to achieve the circuit and all connections thereto.

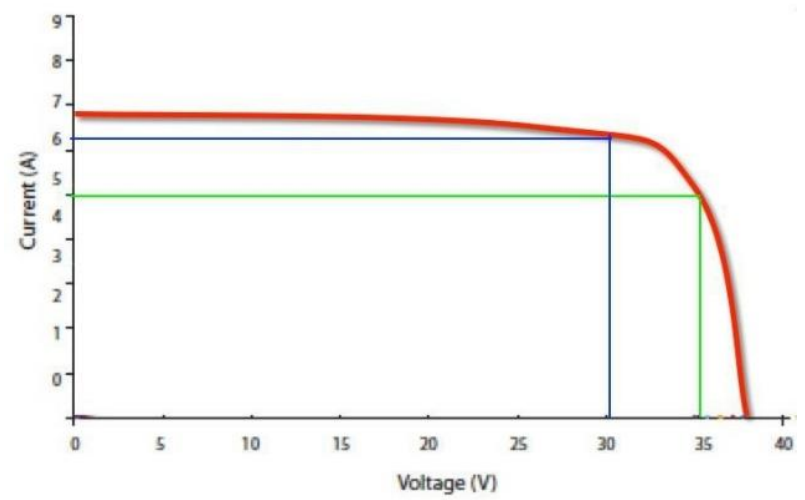

Figure 3. I-V curves of PV panell

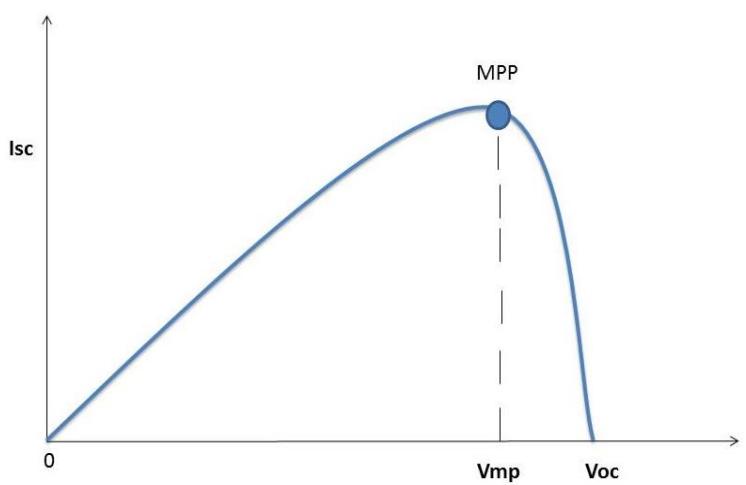

Figure 4. Defining point of MPPT

After obtaining a voltage and a current can charge the battery. Since this is not an ordinary one and it needs a charging principle, in order to obtain the highest efficiency.

To charge a LiFePO4 battery, we need two charging cycles, the first cycle must be in constant current until the battery gets $95 \%$ of its capacity after to be given in the constant voltage charging mode. 
This battery supports only one type of the charging, constant current, but the battery will charge only until it reaches $95 \%$ capacity. Charging mode is represented schematically in (Figure 5). This mode is welcome because it increases efficiency and lifetime of the battery.

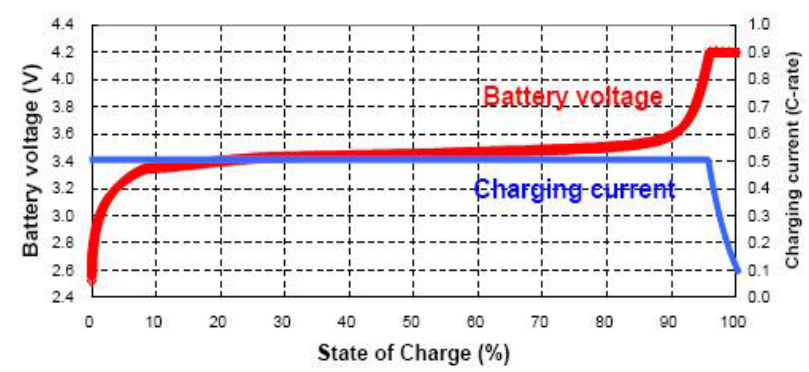

Figure 5. Charging a $\mathrm{LiFePO}_{4}$ battery

As mentioned above, this type of batteries can be discharge to a minimum battery $20-15 \%$ compared to the lead-acid that can be used up to a maximum of $75-70 \%$, which will increase the amount for which batteries can be used.

All data regarding battery capacity at the time of given current voltage on the battery, surface temperature, operating hours, while charging, different types of faults, alerts and other notifications should be sent to a station that monitors all system that can be either in the immediate vicinity, or be at a greater distance.

For this project I used a microcontroller for communication module, battery charging and MPPT system, a Wi-Fi communication module for communication with another module to achieve network and test its functionality (figure 6). I made a bench of batteries with a capacity of $240 \mathrm{~W} / \mathrm{h}$ which was enough lighting at night ( 7 hours) for 2 consecutive days without needing a recharge them (test done in case of rainy days it is not possible while recharging the battery on the day).

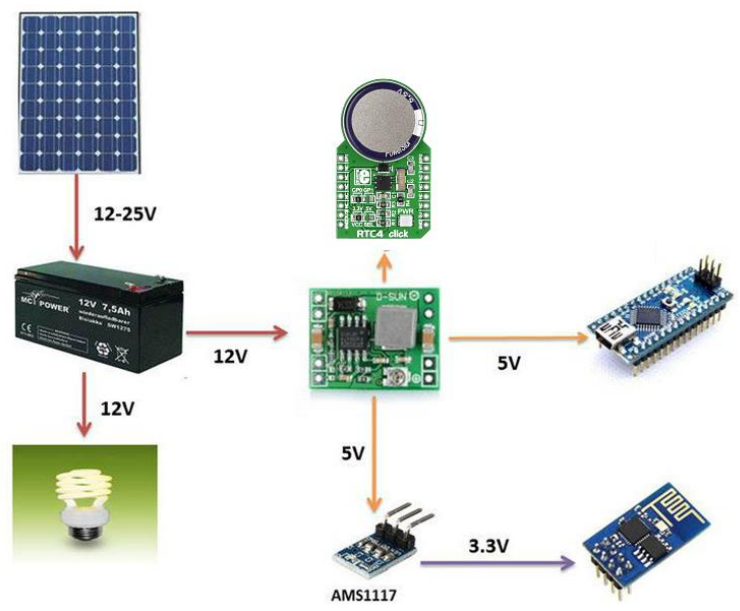

Figure 6. System's components
I made a prototype circuit (Figure 7) to verify the effectiveness and functionality of the proposed system. This prototype can be seen in the image below.

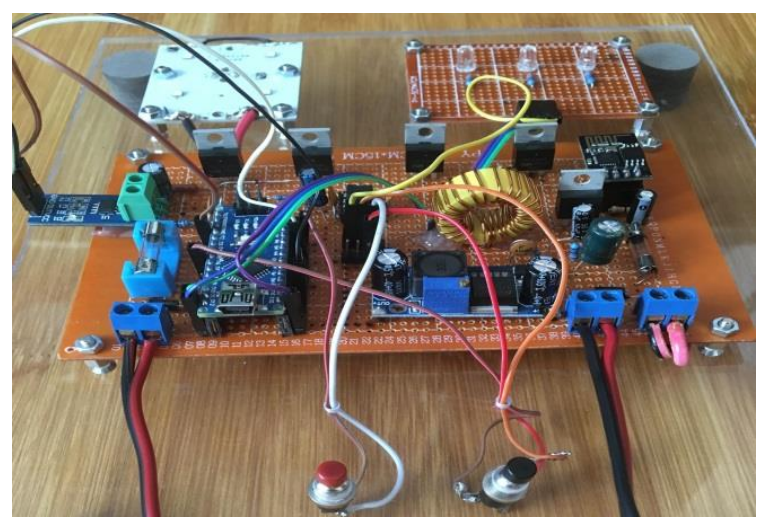

Figure 7. Prototypes

This system can be adopted in the future to make a power supply more power (to longer battery life) and product development in further (regarding low efficiency because of poor parts and the algorithm itself, in the future I intend to make changes in terms of efficiency and remote control to monitor easily and painlessly.

\section{CONCLUSION}

Our intention was directed at this time to produce a prototype allowing the incorporation of modern technologies for lighting control of LED lamps. Control obtained by MPPT proved efficient transmission of system parameters through the Wi-Fi performed properly, command lamp status (on / off) or illumination it through illumination sensor, a real time clock and remote control, every moment had normal errors.

To develop and improve this project can be mounted various sensors such light sensor, presence sensor, astronomical clock to efficient lighting without any losses due to the use battery when daylight or to have a realistic timetable when to start and when to stop lighting without the need to connect or disconnect data transmission luminaires.

Integrating a network of wireless sensors of several lamps, tests coverage areas varying finding solutions to minimize accumulated error of real-time clock and connecting a GPRS module is our research targets in the near future.

\section{REFERENCES}

[1] N. Golovanov, V. Racicovschi, R. Porumb, C. Ivanovici, I. Popa, L.A. Koteleș, S. Crăciun, "Sisteme de iluminat electric cu LED-uri. Eficiența enegetică", 2009.

[2] M. Collota, S. Tirrito, R. Caponetto, "Flexible street lamp lighting system management through Power Line", 2014. 
[3] H.G. Coanda, "Designing a control system for smart outdoor street lighting using advanced communication technologies", The Scientific Bulletin of the Electrical Engineering Faculty, year 2015, no.1 (29), ISSN 1843-6188, pp. 25-30.

[4] C. Jing, D. Shu, D. Gu, "Design of Streetlight Monitoring and Control System Based on Wireless Sensor Networks", Industrial Electronics and Applications, ICIEA. 2nd IEEE Conference on, ISBN: 978-1-4244-0736-1, 2007

[5] O. Natu, S.A. Chavan, "GSM Based Smart Street Light Monitoring and Control System", International Journal on Computer Science and Engineering (IJCSE), ISSN 0975-3397, Vol. 5 No. 03 Mar 2013, pp.187-189.

[6] R. Capponetto, G. Dongola, L. Fortuna, N. Riscica, D. Zufacchi, "Power Consumption Reduction in a Remote Controlled Street Lighting”, Power Electronics, Electrical Drives, Automation and Motion, SPEEDAM International Symposium on, ISBN: 978-1-4244-1663-9, 2008

[7] F. Leccese "Remote-Control System of High Efficiency and Intelligent Street Lighting Using a ZigBee Network of Devices and Sensors", IEEE Transactions On Power Delivery, Vol. 28, No. 1, January 2013, pp.21-28.

[8] F. Leccese, "Remote-Control System of High Efficiency and Intelligent Street Light Using a ZigBee Network of Devices and Sensors", IEEE Transactions on Power Delivery, ISSN: 0885-8977, pp.21-28, 2013

[9] Z. Chen, C. Hu, J. Liao, S. Liu, "Protocol Architecture for Wireless Body Area Network Based on nRF24L01", Shenzhen, China, 2008

[10] W. Yongqing, H. Chuncheng, Z. Suoliang, H. Yali, and W. Hong, "Design of solar LED street lamp automatic control circuit" in Proc. Int. Conf. Energy Environment Technol., Oct. 16-18, 2009, vol. 1, pp. 90-93.

[11] Z. Yao-Lin, Z. Gao-Qiang, Z. Lei, X. Jin, “Design of Wireless Multi-point Temperature Transmission System Based on nRF24L01”, Xi'an, China, 2011 